\title{
e Xactal AvaliaÇÃo dos Parâmetros TermodinÂMICOS E Cinéticos de Cenouras Submetidas a Secagem CONVECTIVA \\ ISSN: 1984-3151
}

\author{
Evaluation of Thermodynamic and Kinetic Parameters of \\ Carrots Submitted to Convective Drying
}

\author{
Heloísa Righi Pessoa da Silva'; Roberta Pazinato Cuco²; Barbara Daniele Almeida \\ Porciuncula ${ }^{3}$; Camila da Silva ${ }^{4}$
}

1 Graduanda de Engenharia de Alimentos. Universidade Estadual de Maringá, 2017. Umuarama/PR. righihelois@gmail.com.

2 Mestranda em Engenharia Química. Universidade Estadual de Maringá, 2017. Graduada em Engenharia de Alimentos, Universidade Estadual de Maringá. Umuarama, PR. cucoroberta@gmail.com.

3 Doutora em Engenharia de Alimentos. Universidade Federal de Santa Catarina, 2014. Professora do Departamento de Tecnologia. Universidade Estadual de Maringá, Umuarama, PR. barbara.porciuncula@gmail.com.

4 Doutora em Engenharia Química. Universidade Estadual de Maringá, 2009. Professora do Departamento de Tecnologia. Universidade Estadual de Maringá, Umuarama, PR. camiladasilva.eq@gmail.com.

Recebido em: 02/04/2017 - Aprovado em: 20/09/2017 - Disponibilizado em: 30/11/2017

RESUMO: Este estudo tem como objetivo a obtenção de dados cinéticos da secagem de cenouras, bem como o ajuste de modelos matemáticos aos dados experimentais e determinação dos parâmetros termodinâmicos do processo. Buscando este propósito, experimentos foram conduzidos em estufa com renovação e circulação de ar, nas temperaturas de 50,60,70 e $80^{\circ} \mathrm{C}$. Os modelos matemáticos foram ajustados aos dados das cinéticas de secagem à diferentes temperaturas, e os modelos de Page e de Midilli apresentaram o maior coeficiente de correlação $\left(R^{2}\right)$ e menor valor de RMSE. Da análise termodinâmica verificou-se que a entalpia e a energia livre de Gibbs diminuíram com o aumento da temperatura, característico de um processo endotérmico. A entropia apresentou valores negativos e diminuiu com a diminuição na temperatura de secagem, isto indica que há um aumento no grau de ordem do sistema, considerado desfavorável entropicamente.

PALAVRAS-CHAVE: Secagem. Cenouras. Parâmetros termodinâmicos.

ABSTRACT: This study aims to obtain kinetic data of carrot drying, as well as the adjustment of mathematical models to the experimental data and determination of the thermodynamic parameters of the process. For this purpose, experiments were conducted in an oven with renovation and circulation of air, at temperatures of 50, 60, 70 and 80 ${ }^{\circ} \mathrm{C}$. The mathematical models were adjusted to the drying kinetics data at different temperatures, and the Page and de Midilli models presented the highest correlation coefficient $\left(R^{2}\right)$ and lowest RMSE values. From the thermodynamic analysis it was verified that the enthalpy and the free energy of Gibbs decreased with the increase of the temperature, characteristic of an endothermic process. The entropy presented negative values and decreased with the decrease in the drying temperature, this indicates that there is an increase in the degree of order of the system, considered as entropically unfavorable.

KEYWORDS: Drying. Carrots. Thermodynamic parameters. 


\section{INTRODUÇÃo}

Raízes de cenoura (Daucus carota L.) possuem alto valor nutritivo, são uma fonte de provitamina, a um baixo custo (ROSA, 2010). Devido a isso, observa-se o aumento da utilização da cenoura como matériaprima na indústria de alimentos para a obtenção de uma ampla variedade de produtos (ROSA, 2010, SILVA, 2003).

Os pigmentos responsáveis pela cor de cenoura são os chamados carotenoides, sendo que o $\beta$-caroteno constitui uma grande porção destes carotenoides (OWOLADE et al., 2017). O $\beta$-caroteno é uma vitamina lipossolúvel que possui atividades biológicas importantes destacando-se a prevenção de doenças inflamatórias e neurodegenerativas (BANZATTO; FREITA; MUTTON, 2013, OLIVEIRA et al., 2017) além de propriedades antioxidantes (WANG et al., 2017). No entanto, eles são extremamente sensíveis à exposição à luz, ao calor, ao oxigênio e aos ácidos (BARROS; GRIMALDI; CABRAL, 2017).

Em consequência da instabilidade, na maioria das vezes, o processamento do alimento causa um percentual de degradação dos carotenoides (GAMA; SYLOS, 2007). Estudos realizados por Qiu, Chen e Li (2009) analisaram que a concentração de oxigênio exerce maior influência na estabilidade do $\beta$-caroteno do que as temperaturas de aquecimento.

A secagem ao ar é um dos métodos fundamentais de processamento de alimentos pois, devido, à redução da atividade de água, aumenta a segurança dos alimentos e prolonga a sua vida útil (DOYMAZ, 2006, KOWALSKI; SZADZIŃSKA; LECHTAŃSKA, 2013). A remoção de água visa prevenir o desenvolvimento de um ambiente favorável ao crescimento de microrganismos (KOWALSKI; SZADZIŃSKA; LECHTAŃSKA., 2013). A técnica de secagem é baseada na transferência de um líquido que está num sólido úmido para uma fase gasosa não saturada devido à diferença de temperatura entre os meios (FOUST et al., 2012).
Os fenômenos ocorridos durante a secagem são muitos complexos, desta forma existem diversas teorias e fórmulas para descrever a taxa de secagem, uma delas é a teoria difusional (PARK; YADO; BROD, 2001). Em processos difusionais um dos modelos mais utilizados para descrever a transferência de massa é o de Fick (CREMASCO, 2008). Além do modelo de Fick para descrever 0 processo de secagem existem modelos semi-teóricos e modelos empíricos. Os modelos semi-teóricos são derivados das soluções gerais da Lei de Fick. Os modelos empíricos não têm fundamentação teórica, mas são baseados em dados experimentais e estatísticos, que permitem prever e simular o comportamento de alguns parâmetros da cinética de secagem de forma simples e com fácil aplicação. (LISBÔA et al., 2015). Alguns modelos bastante utilizados são o modelo de Page, modelo de Handerson e Pabis, modelo de Lewis, Modelo de Wang e Singh e modelo de Thompson, Logaritmo, Midilli, Newton, Peleg, Twoterm e Twotermexp. (YALDIZ; ERTEKI; UZUN, 2001; MIDILLI; KUCUK; YAPAR, 2002; BABALIS et al., 2006).

O estudo de propriedades termodinâmicas nos processos de secagem é fundamental para compreensão e avaliação das condições de processos industriais, sobre as trocas de energia que ocorrem a partir de um estado de equilíbrio para o outro e solucionar alguns parâmetros de estabilidade e otimização (COSTA et al., 2016).

Com base nesse contexto, o presente trabalho teve como objetivo a obtenção de dados cinéticos da secagem de cenouras, bem como o ajuste de modelos matemáticos aos dados experimentais e determinação dos parâmetros termodinâmicos do processo. 


\section{MATERIAIS e MÉTOdOS}

\subsection{Preparo e Caracterização da Matéria- Prima}

Foram utilizadas cenouras adquiridas no comércio local do município de Umuarama, Paraná. A seleção das amostras foi realizada pelo grau de maturação das mesmas, observado por análise visual e pelo teor de sólidos solúveis ( $\left.{ }^{\circ} \mathrm{Brix}\right)$. As cenouras foram lavadas em água corrente e trituradas em um multiprocessador (WALITA, RI7625). Para a determinação do teor de sólidos solúveis utilizou-se um refratômetro (REICHERT) calibrado em ${ }^{\circ}$ Brix com água destilada. Com o auxílio de uma gaze comprimiu a cenoura triturada até que ela soltasse o sumo. O teor de umidade das cenouras in natura foi determinado utilizando o método gavimétrico.

\subsection{SeCAgem}

As amostras de cenoura trituradas foram secas em estufa com circulação e renovação de ar (MARCONI MA035). Aproximadamente $25 \mathrm{~g}$ de amostra foram acondicionadas em placas de Petri e secas nas temperaturas de $50,60,70$ e $80{ }^{\circ} \mathrm{C}$, até que se atingisse peso constante. A umidade relativa (UR) foi monitorada, de hora em hora, pelas medidas de bulbo úmido e seco. Para manter a uniformidade do leito de secagem, as amostras foram comprimidas com uma força de 9,8 $\mathrm{N}$ por 10 segundos. As amostras iniciaram o processo de secagem com espessura de $4,0 \pm 0,2 \mathrm{~mm}$. Foram realizadas pesagens a cada 15 minutos durante as 2 primeiras horas de processos e a cada 30 minutos até que as mesmas atingissem peso constante.

\subsection{Ajuste dos Modelos Matemáticos}

Para o ajuste dos modelos matemáticos aos dados experimentais de secagem, realizou-se uma análise de regressão não linear de nove modelos, apresentados na Tabela 1, utilizando-se o programa computacional MATLAB R2010a. O critério de escolha do melhor modelo cinético foi baseado nos valores do coeficiente de determinação $\left(R^{2}\right)$ e nos valores da raiz do erro médio quadrático (RMSE), mostrados na Tabela 2.

\subsection{ANÁLISE TERMOdINÂMICA}

Para determinação dos dados termodinâmicos a constante de secagem $(k)$ dos melhores modelos matemáticos ajustados foram aplicadas a equação de Arhenius, que mostra a relação entre a energia de ativação e a velocidade em que o processo ocorrre. Foram determinadas as propriedades termodinâmicas, entalpia $(\Delta \mathrm{H})$, entropia $(\Delta S)$ e energia de Gibbs $(\Delta \mathrm{G})$ pelo método descrito por Jideani e Mpotokwana (2009).

Tabela 1 - Modelos matemáticos aplicados às curvas de secagem

\begin{tabular}{ccc}
$\begin{array}{c}\text { Número do } \\
\text { Modelo }\end{array}$ & Equação & $\begin{array}{c}\text { Modelo (MIDILLI; KUCUK; YAPAR, } \\
\text { 2002, YALDIZ; ERTEKI; UZUN, 2001) }\end{array}$ \\
\hline 1 & $\mathrm{f}(\mathrm{x})=\mathrm{a}^{*} \exp \left(-\mathrm{k}^{*} \mathrm{x}\right)$ & Henderson e Pabis \\
2 & $\mathrm{f}(\mathrm{x})=\mathrm{a}^{*} \exp \left(-\mathrm{k}^{*} \mathrm{x}\right)+\mathrm{c}$ & Logaritmo \\
3 & $\mathrm{f}(\mathrm{x})=\exp \left(-\mathrm{k}^{*} \mathrm{x}^{\wedge} \mathrm{n}\right)+\mathrm{b}^{*} \mathrm{x}$ & Midilli \\
4 & $\mathrm{f}(\mathrm{x})=\exp \left(-\mathrm{k}^{*} \mathrm{x}\right)$ & Newton \\
5 & $\mathrm{f}(\mathrm{x})=\exp \left(-\mathrm{k}^{*} \mathrm{x}^{\wedge} \mathrm{n}\right)$ & Page \\
6 & $\mathrm{f}(\mathrm{x})=1-\left(\left(\mathrm{x} /\left(\mathrm{k}_{1}+\mathrm{k}^{*} \mathrm{x}\right)\right)^{*} 1 / \mathrm{X}_{0}\right)$ & Peleg \\
7 & $\mathrm{f}(\mathrm{x})=\mathrm{a}^{*} \exp \left(-\mathrm{k}^{*} \mathrm{x}\right)+\mathrm{b}^{*} \exp \left(-\mathrm{k}_{1}{ }^{*} \mathrm{x}\right)$ & Twoterm \\
8 & $\mathrm{f}(\mathrm{x})=\mathrm{a}^{*} \exp \left(-\mathrm{k}^{*} \mathrm{x}\right)+(1-\mathrm{a})^{*} \exp \left(-\mathrm{k}^{*} \mathrm{a}^{*} \mathrm{x}\right)$ & Twotermexp \\
9 & $\mathrm{f}(\mathrm{x})=1+\left(\mathrm{a}^{*} \mathrm{x}\right)+\left(\mathrm{b}^{*} \mathrm{x}\right)^{\wedge} 2$ & Wang e Sing \\
\hline
\end{tabular}

${ }^{*} \mathrm{k}, \mathrm{a}, \mathrm{b}, \mathrm{c}, \mathrm{n}, \mathrm{k}_{0}$ e $\mathrm{k}_{1}$ são constantes dos modelos; $\mathrm{x}$ é o tempo de secagem (h) e $\mathrm{X}_{0}$ é a umidade inicial de cada temperatura. Fonte - Próprio autor. 
Tabela 2 - Dados do coeficiente de determinação e raiz do erro médio quadrático para os diferentes modelos avaliados

\begin{tabular}{ccccccccc}
\hline & \multicolumn{2}{c}{$50^{\circ} \mathrm{C}$} & \multicolumn{2}{c}{$60{ }^{\circ} \mathrm{C}$} & \multicolumn{2}{c}{$70{ }^{\circ} \mathrm{C}$} & \multicolumn{2}{c}{$8{ }^{\circ} \mathrm{C}$} \\
Modelos & $\mathrm{R}^{2}$ & $\mathrm{RMSE}$ & $\mathrm{R}^{2}$ & $\mathrm{RMSE}$ & $\mathrm{R}^{2}$ & $\mathrm{RMSE}$ & $\mathrm{R}^{2}$ & $\mathrm{RMSE}$ \\
\hline 1 & 0.962 & 0,072 & 0,963 & 0,072 & 0,960 & 0,076 & 0,956 & 0,079 \\
2 & 0,989 & 0,038 & 0,985 & 0,047 & 0,982 & 0,052 & 0,982 & 0,053 \\
3 & 0,996 & 0,022 & 0,995 & 0,026 & 0,996 & 0,024 & 0,995 & 0,028 \\
4 & 0,948 & 0,083 & 0,950 & 0,081 & 0,945 & 0,085 & 0,945 & 0,084 \\
5 & 0,994 & 0,029 & 0,993 & 0,029 & 0,995 & 0,026 & 0,994 & 0,030 \\
6 & 0,984 & 0,046 & 0,976 & 0,057 & 0,971 & 0,065 & 0,974 & 0,061 \\
7 & 0,972 & 0,066 & 0,983 & 0,051 & 0,977 & 0,063 & 0,968 & 0,076 \\
8 & 0,988 & 0,040 & 0,988 & 0,041 & 0,989 & 0,040 & 0,986 & 0,045 \\
9 & 0,993 & 0,031 & 0,992 & 0,032 & 0,988 & 0,040 & 0,989 & 0,039 \\
\hline
\end{tabular}

Fonte - Próprio autor.

\section{ReSULtAdOS E Discussão}

\subsection{Modelos Matemáticos}

O teor de umidade e o teor de sólidos solúveis para as amostras in natura são de $90,30 \pm 0,88 \%$ e $8,15 \pm 0,49$ ${ }^{\circ}$ Brix à $26,02 \pm 0,79^{\circ} \mathrm{C}$.

Os modelos apresentados na Tabela 1 foram ajustados aos dados experimentais das cinéticas de secagem da cenoura a diferentes temperaturas. A Tabela 2 demonstra os valores do coeficiente de determinação $\left(R^{2}\right)$ e raiz do erro médio quadrático (RMSE) para os 9 modelos avaliados neste trabalho.

Analisando os dados apresentados na Tabela 2, verifica-se que os modelos que obtiveram melhores ajustes aos dados experimentais nas temperaturas avaliadas foram os modelos 3 e 5 descritos na Tabela 1 , uma vez que estes apresentaram o maior $R^{2} e$ menor valor do RMSE. O ajuste destes modelos aos dados experimentais é apresentado na Figura 1.

Pelos dados apresentados na Figura 1, nota-se que a temperatura exerce influência sobre a velocidade de secagem, quanto maior a temperatura maior é a taxa de secagem das amostras.

Observa-se também que a temperatura de $80{ }^{\circ} \mathrm{C}$ apresentou menor umidade final e menor tempo de secagem. Isso ocorre, pois, com o aumento da temperatura, ocorre maior diferença de temperaturas entre a amostra e o ar de secagem, ocasionando maior transferência de calor (TONON; BRABET; HUBINGER, 2009). Pinedo e Murr (2005) reportaram resultados similares ao secarem cenouras em secador a vácuo.

Através da Figura 1 pode-se observar também que os modelos de Page e Midilli apresentaram bom ajuste aos valores experimentais de amostras de cenoura. Existem relatos na literatura que mostram que o modelo de Midilli é adequado para descrever curvas de secagem de folhas de endro e de salsinha (DOYMAZ, 2006), assim como o modelo de Page para descrever curvas de secagem de kiwis (SIMAL; FEMENIA; GARAU, 2005), feijão, batata e ervilha (SENADEERA et al., 2003). Na Tabela 3 estão apresentados os parâmetros de ajuste dos modelos aos dados experimentais. Observa-se que os valores de $\mathrm{k}$ aumentaram com o aumento da temperatura de secagem, isto se deve, pois o parâmetro $k$ é a constante de secagem, utilizada para caracterizar o efeito da temperatura (BABALIS; BELESSIOTIS, 2004). Estudos de modelagem matemática durante a secagem de materiais biológicos observaram comportamento semelhante da constante de 
secagem, crescente com a temperatura (AZEEZ et al., 2017; BABALIS; BELESSIOTIS, 2004). Pode-se notar também que o parâmetro $\mathrm{n}$ em ambos os modelos, apresentou comportamento ascendente com a temperatura, porém muito semelhantes entre si, o que sugere que conforme discutido por Simpson et al. (2017), este parâmetro depende da microestrutura do material e não da temperatura.

Figura 1 - Ajustes do modelo de Page e Midilli

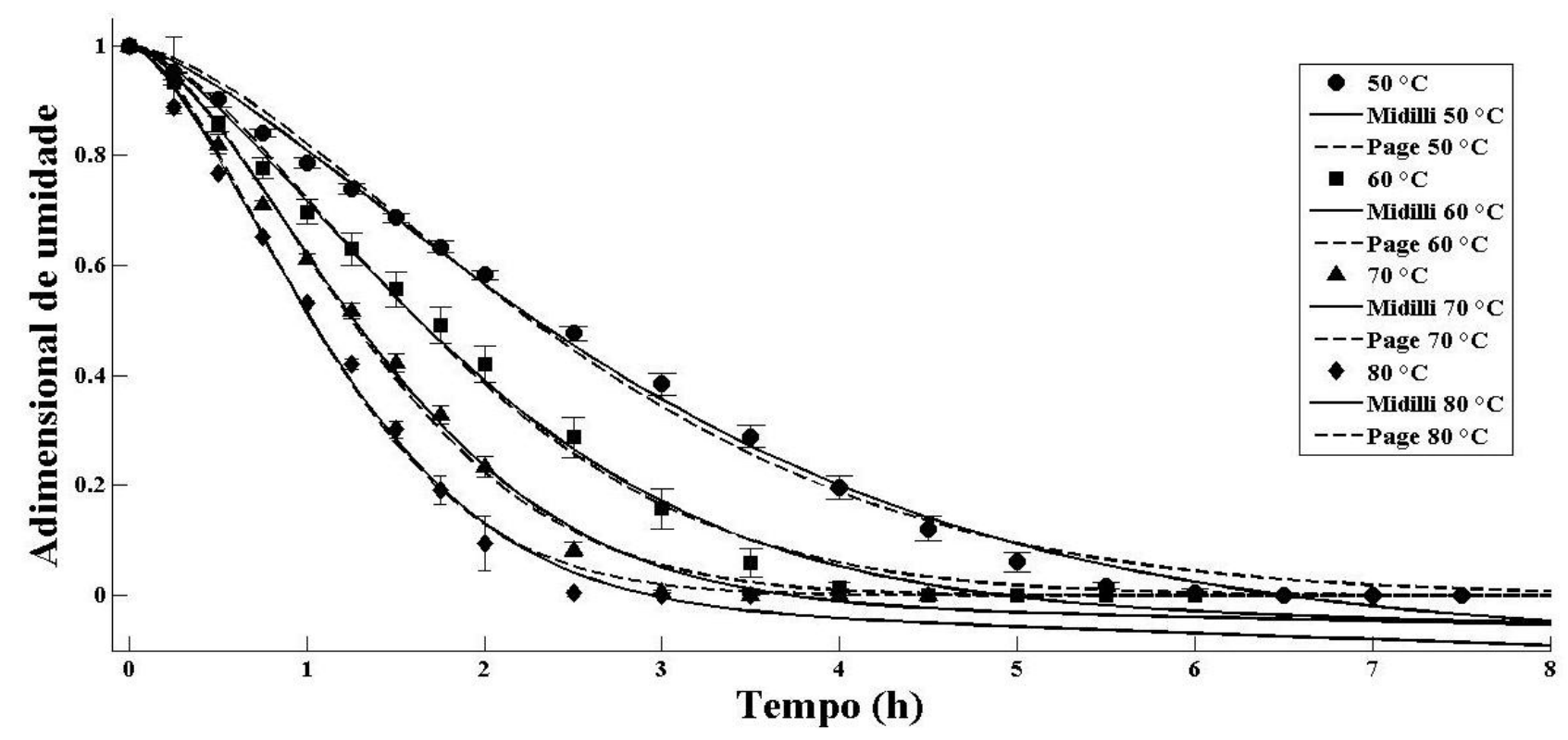

Fonte - Próprio autor.

Tabela 3 - Valores das constantes empíricas dos modelos de Page e Midilli

\begin{tabular}{cccccc}
\hline $\mathrm{T}\left({ }^{\circ} \mathrm{C}\right)$ & & Midilli & \multicolumn{3}{c}{ Page } \\
\hline $\mathbf{5 0}$ & $\mathbf{b}$ & $\mathbf{k}$ & $\mathbf{n}$ & $\mathbf{k}$ & $\mathbf{n}$ \\
$\mathbf{6 0}$ & $-0,01$ & 0,20 & 1,42 & 0,20 & 1,54 \\
$\mathbf{7 0}$ & $-0,01$ & 0,33 & 1,48 & 0,33 & 1,56 \\
$\mathbf{8 0}$ & $-0,01$ & 0,47 & 1,58 & 0,48 & 1,63 \\
\hline
\end{tabular}

Fonte - Próprio autor.

\subsection{ANÁLISE TERMODINÂMICA}

O modelo de Midilli apresentou uma energia de ativação $36520,08\left(\mathrm{~J} \cdot \mathrm{mol}^{-1} \cdot \mathrm{K}^{-1}\right)$ menor do que 0 modelo de Page 38007,45 (J.mol-1. $\left.\mathrm{K}^{-1}\right)$. Em processos de secagem quanto menor for a energia de ativação maior será a difusividade de água no produto, isto é mais baixa será a energia necessária para transformar a água em vapor (CORRÊA et al., 2010).

$\mathrm{Na}$ Tabela 4 estão apresentados os valores dos parâmetros termodinâmicos para o modelo de Midilli e de Page. Observando os valores de entalpia notou-se que ela diminui com o aumento da temperatura, indicando que é necessária uma menor quantidade de energia para a secagem ocorrer em temperaturas mais altas, indicando também que o processo é endotérmico. Costa et al. (2016) observaram esse mesmo efeito durante a secagem de casca de jabuticaba.

e-xacta, Belo Horizonte, v. 10, n. 2, p. 73-80. (2017). Editora UniBH doi: $10.18674 /$ exacta.v10i2.2171 
Tabela 4 - Parâmetros termodinâmicos para o modelo de Midilli e de Page.

\begin{tabular}{ccccccc}
\hline \multicolumn{5}{c}{ Midilli } & \multicolumn{3}{c}{ Page } \\
\hline $\mathrm{T}\left({ }^{\circ} \mathrm{C}\right)$ & $\Delta \mathrm{H}\left(\mathrm{kJ}^{\mathrm{mol}}{ }^{-1}\right)$ & $\Delta \mathrm{S}\left(\mathrm{J} \cdot \mathrm{mol}^{-1} \cdot \mathrm{K}^{-1}\right)$ & $\Delta \mathrm{G}\left(\mathrm{kJ}_{\mathrm{mol}}^{-1}\right)$ & $\Delta \mathrm{H}\left(\mathrm{kJ} \cdot \mathrm{mol}^{-1}\right)$ & $\Delta \mathrm{S}\left(\mathrm{J}_{\mathrm{mol}}^{-1} \cdot \mathrm{K}^{-1}\right)$ & $\Delta \mathrm{G}\left(\mathrm{kJ} \cdot \mathrm{mol}^{-1}\right)$ \\
\hline 50 & 33,83 & $-145,55$ & 80,85 & 35,32 & $-141,01$ & 80,87 \\
60 & 33,75 & $-145,81$ & 82,31 & 35,24 & $-141,26$ & 82,28 \\
70 & 33,67 & $-146,05$ & 83,76 & 35,16 & $-141,51$ & 83,69 \\
80 & 33,59 & $-146,29$ & 85,23 & 35,07 & $-141,75$ & 85,11 \\
\hline
\end{tabular}

Fonte - Próprio autor.

A entropia é uma grandeza termodinâmica associada ao grau de desordem de um sistema. Neste estudo a entropia apresentou valores negativos e diminuiu com o aumento da temperatura de secagem, isto indica que há um aumento no grau de ordem do sistema, considerado desfavorável entropicamente. Jideani e Mpotokwana (2009) observaram efeito semelhante, durante um processo de absorção de água em bananas. Esse fato é esperado, pois com o aumento da temperatura aumenta o grau de excitação das moléculas, resultando num aumento da desordem do sistema (CORRÊA et al., 2010). De acordo com a teoria do complexo ativado, uma substância num estado de ativação só pode ter entropia negativa se graus de liberdade de translação ou rotação são perdidos durante a formação do complexo ativado (JIDEANI; MPOTOKWANA, 2009).

A energia livre de Gibbs pode ser considerada como uma medição do trabalho realizado pelo sistema, neste estudo apresentou resultados positivos que aumenta com a temperatura, indicando que a secagem não foi espontânea (CORRÊA et al., 2010).
O valor positivio é característico de um processo endergônico, em que requer adição de energia externa para que o processo ocorra (COSTA et al., 2016; JIDEANI; MPOTOKWANA, 2009). Corrêa et al. (2010) e Costa et al. (2016) encontraram valores positivos e crescentes da energia livre de Gibbs com o aumento da temperatura, durante o processo de secagem.

\section{ConcLusão}

De acordo com os resultados obtidos no presente estudo pode-se afirmar que 0 uso de modelos cinéticos são uma alternativa para prever os aspectos de secagem a diferentes temperaturas, sendo observado a correspondência entre os valores experimentais e os modelos de Page e Midilli. Ao realizar análise termodinâmica verificou-se que a entalpia e a energia livre de Gibbs diminuíram com o aumento da temperatura mostrando que o processo é endotérmica, enquanto a entropia também decresceu e foi negativa, sendo considerado um fator desfavorável entropicamente.

\section{REFERÊNCIAS}

AZEEZ, L. et al.. Bioactive compounds' contents, drying kinetics and mathematical modelling of tomato slices influenced by drying temperatures and time. Journal of the Saudi of Agricultural Sciences,
2017. Disponível em: <http://dx.doi.org/10.1016/j.jssas.2017.03.002> Acesso: 25 março 2017. 
BABALIS, S. J.; BELESSIOTIS, V. G. Influence of the drying conditions on the drying constants and moisture diffusivity during the thin-layer drying of figs. Journal of Food Engineering, v.65, n. 3, 449-458, 2004.

BABALIS, S. J. et al. Evaluation of thin-layer drying models for describing drying of figs (Ficus carica). Journal of Food Engineering. v. 75, 205-214, 2006.

BANZATTO, D.; FREITA, L.A.; MUTTON, M. J. R. Carotenoid production by Rhodotorula rubra cultivated in sugarcane juice, molasses, and syrup. Ciência e Tecnologia de Alimentos. V.33, 14-18, 2013.

BARROS, H. D. F. Q.; GRIMALDI, R.; CABRAL, F. A. Lycopene-rich avocado oil obtained by simultaneous supercritical extraction from avocado pulp and tomato pomace. The Journal of Supercritical Fluids, v. 120, 1-6, 2017.

CORRÊA, G. C. et al. Modelagem matemática e determinação das propriedades termodinâmicas do café (Coffea arabica L.) durante o processo de secagem. Revista Ceres, v.57, 595-601, 2010.

COSTA, C. F. et al. Mathematical modeling and determination of thermodynamic properties of jabuticaba peel during the drying process. Revista Brasileira de Engenharia Agrícola e Ambiental, v.20, $576-580,2016$.

CREMASCO, M. A. Fundamentos de Transferência de Massa. Campinas: UNICAMP, 725 p., 2002, ISBN 85-268-0595-9.

DOYMAZ, I. Thin-layer drying behaviour of mint leaves. Journal Food Engineering, v. 74, 370-375, 2006.

FOUST, A. S. et al.. Princípios das operações unitárias. Rio de Janeiro: Editora LTC, 670 p., 2012, ISBN 85-216-1038-6.

GAMA, J. J. T.; SYLOS, C. M. Effect of thermal pasteurization and concentration on carotenoid composition of brazilian valencia orange juice. Food Chemistry, v. 100, n. 4, 1686-1690, 2007.

JIDEANI, V. A.; MPOTOKWANA, S. M. Modeling of water absorption of botswana bambara varieties using Peleg's equation. Journal of Food Engineering, v.92, 182-188, 2009.

KOWALSKI, S. J.; SZADZIŃSKA, J.; LECHTAŃSKA, J. Non-stationary drying of carrot: Effect on product quality. Journal Food Engineering, v. 118, 393-399, 2013.
LISBÔA, J. F. et al.. Análise da Hidratação de grãos de alpiste. Revista Brasileira de Engenharia Agrícola e Ambiental, v. 19, 218-223, 2015.

MIDILLI, A.; KUCUK, H.; YAPAR, Z. A new model for single-layer drying. Drying Technology, v. 20, 15031513, 2002.

OLIVEIRA, $\mathrm{H}$ et al.. Gastrointestinal absorption, antiproliferative and anti-inflammatory effect of the major carotenoids of: Gardenia jasminoides Ellis on cancer cells . Food and Function, v. 8, 1672-1679, 2017.

OWOLADE, S.O. et al.. Study on physico-chemical properties, antioxidant activity and shelf stability of carrot (Daucus carota) and pineapple (Ananas comosus) juice blend. International Food Research Journal, v. 24, 534-540, 2017.

PARK, K. J.; YADO, M. K. M.; BROD, F. P. R. Estudo de secagem de pêra bartlett (pyrus sp.) em fatias. Ciência e Tecnologia de Alimentos. v. 21, 288-292, 2001.

PINEDO, A. A.; MURR, F. E. X. Influência da pressão, temperatura e pré-tratamentos na secagem a vácuo de cenoura e abóbora. Ciência e Tecnologia de Alimentos, 36-643, 2005.

QIU, D.; CHEN, Z. R.; LI, H. R. Effect of heating on solid $\beta$-carotene. Food Chemistry, v. 112, 344-349, 2009.

ROSA, J. G. Secagem da cenoura (Daucus carota L.) em micro-ondas. 78 f. Dissertação de Mestrado de Engenharia Química (Pesquisa e Desenvolvimento de Processos Químicos) - Universidade Federal de São Carlos, São Carlos, 2010.

SENADEERA, W. et al.. Influence of shapes of selected vegetable materials on drying kinetics during fluidized bed drying. Journal of Food Engineering, v.58, 277-283, 2003.

SILVA, E. G. Composição bioativa de cenoura em função da época de colheita e do processamento mínimo. 75 f. Dissertação de Mestrado em Engenharia Alimentar (Qualidade e Segurança Alimentar) - Instituto Superior de Agronomia, Universidade Técnica de Lisboa, Lisboa, 2003.

SIMAL, S.; FEMENIA, A.; GARAU, C. R. Use of exponential, Page's and diffusional models to simulate the dying kinetics of kiwi fruit. Journal of Food Engineering, v. 66, 323-328, 2005. 
SIMPSON, R. et al.. Understanding the success of Page's model and related empirical equations in fitting experimental data of diffusion phenomena in food matrices. Trends in Food Science e Technology. v. 62, 194-201, 2017.

TONON, R. V.; BRABET, C.; HUBINGER, M. D. Influência da temperatura do ar de secagem e da concentração de agente carreador sobre as propriedades físico-químicas do suco de açaí em pó. Ciência e Tecnologia de Alimentos, v. 29, 444-450, 2009.
WANG, X. et al.. Supercritical carbon dioxide extraction of $\beta$-carotene and and $\alpha$-tocopherol from pumpkin: a Box-Behnken design for extraction variables. Analytical Methods, v.9, 294-303, 2017.

YALDIZ, O.; ERTEKI, C.; UZUN, H. I. Mathematical modeling of thin layer solar drying of sultana grapes. Energy, v. 26, 457-465, 2001. 